Canadian Oncology

Nursing Journal

Revue canadienne

de soins infirmiers

en oncologie

Volume 27, Issue 4 • Fall 2017

elSSN: 2368-8076 


\title{
Dépistage systématique de la détresse : portraits et regards croisés sur les perceptions des différents acteurs, perspectives cliniques et de recherche Partie 2
}

\author{
par Nicole Tremblay, Odette Roy et Aurélie Lecocq, avec la collaboration de Caroline Provencher, Louise Compagna, Francine \\ Grondin, Karine Le Breton, Jocelyne Doucet et Cécile Mathurin
}

\section{INTRODUCTION}

D ans la première phase de notre étude portant sur le dépistage de la détresse auprès de personnes atteintes de cancers du sein et hématologiques, phase qui constitue le sujet de la partie 1 de cet article (Tremblay et al., 2017, nous avons brossé les portraits de la détresse de ces deux groupes de participants atteints de cancer. Nous avons ainsi mis en évidence que bien que près de $34 \%(33,9 \%)$ de notre échantillon ( $\mathrm{N}=532$ participants) atteignent le score clinique de 5 et plus sur le thermomètre de la détresse (TD), un faible pourcentage d'entre eux $(22,7 \%)$ indique qu'ils souhaitent recevoir de l'aide pour les problèmes relevés lors du dépistage. Et ce pourcentage plutôt faible baisse encore plus au temps $2(14,7 \%)$. Or, un tel score au TD justifie une évaluation plus ciblée de cette détresse. Il en est de même des résultats suivants : un peu plus d'un patient sur 4 (26,6\%) présente à l'échelle d'évaluation des symptômes d'Edmonton (ESAS) un score supérieur ou égal à 5 pour l'anxiété au temps 1 et $18,4 \%$ au temps 2 . Enfin, concernant la dépression, on constate que 9,4 \% des patients ont un score supérieur ou égal à 5 au temps 1 et 9,5\% au temps 2 . Ces situations appellent donc à une évaluation plus poussée, généralement réalisée par un oncopsychologue.

\section{AU SUJET DES AUTEURES}

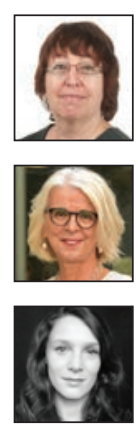

Nicole Tremblay, inf. M.Sc, CSIO (C), ICSP (C), chercheure principale

Odette Roy, inf., M.Sc., M.A.P., Ph.D, co-chercheure

Aurélie Lecocq, (Ph.D) en éducation, psychométricienne chez Assessment strategies inc

Avec la collaboration de

Caroline Provencher, inf. M.Sc.

Louise Compagna, inf. B.Sc., CSIO (C)

Francine Grondin, inf. B.Sc.

Karine Le Breton, inf. M.Sc., CSIO (C)

Jocelyne Doucet, inf. B.Sc., assistante de recherche

Cécile Mathurin, inf. M.Sc., assistante de recherche

DOI: $10.5737 / 23688076274316327$
On a également noté que les participants à l'étude acceptent davantage l'aide offerte lorsque des références leur sont proposées à travers la discussion suivant la prise en compte des résultats de l'outil de dépistage de la détresse (ODD) (40\% des cas au temps 1 et $33 \%$ des cas au temps 2). Ce qui a une importance certaine pour la clinique dont la pratique infirmière. De même, certains temps de la trajectoire de soins sont plus favorables à une acceptation de l'aide offerte telles la période de traitement et l'admission à l'unité d'hémato-oncologie greffe).

Autre résultat important : au temps 2, la proportion de patients ayant un score supérieur ou égal à 5 au TD baisse à $28,1 \%$, mais cette baisse survient peu importe si l'aide offerte a été acceptée ou non, hormis pour les problèmes sociaux où le niveau de détresse baisse significativement si le patient a accepté l'aide offerte. Ces résultats peuvent tout autant indiquer la capacité de résilience de la clientèle atteinte de cancer tout comme les bénéfices des interventions de soutien et d'enseignement principalement réalisées par les infirmières et qui sont souvent peu documentées (De Marinis, et al., 2010; Tremblay et al., 2017).

Ces divers phénomènes décrits dans la première phase de l'étude justifient notre grand intérêt à mieux comprendre les divers enjeux relationnels qui peuvent expliquer ces résultats, notamment les perceptions de la clientèle et les perceptions infirmières à l'égard du dépistage systématique de la détresse afin d'en explorer la teneur, les points de convergence et de divergence entre eux. La suite de cet article traitera donc des résultats de la deuxième phase de cette étude qui porte sur ces perceptions.

\section{MÉTHODOLOGIE \\ Devis}

Tel que nous l'avons décrit lors de la partie I de l'article, un devis mixte séquentiel exploratoire a été développé et utilisé afin de capturer des réalités complexes, telles que des interactions infirmières-patients portant sur un sujet délicat, dans un contexte nouveau et imprévisible (Foss et Ellefsen, 2002).

\section{Objectifs de l'étude}

Cette étude a poursuivi cinq objectifs distincts. Cet article présente les résultats ayant trait aux objectifs 3 et 5 et en partie l'objectif 4. Les objectifs ont été décrits à partir des populations à l'étude. Les trois premiers sont identiques pour les 
deux clientèles atteintes de cancer. Le quatrième objectif vise spécifiquement la population de cancers hématologiques et de greffe, tandis que le cinquième, la population de cancer du sein.

Populations cancers hématologiques et cancer du sein Les objectifs sont de :

1. Recueillir des informations sur la détresse vécue par la clientèle ciblée à des moments précis de la trajectoire de soins; (phase 1)

2. Collecter des informations sur le processus d'implantation du dépistage systématique de la détresse au sein des différents secteurs touchés par le dépistage; (phases 1 à 3)

3. Mieux connaître les perceptions infirmières à l'égard du dépistage systématique de la détresse en contexte de cancers hématologiques, de greffe de cellules hématopoïétiques et de cancer du sein; (phase 2)

En ce qui a trait au 4 e objectif, il a été décrit comme suit :

4. Mieux connaître la perspective du patient à l'égard du dépistage systématique de la détresse en contexte de cancers hématologiques et de greffe de cellules hématopoiétiques; (phase 2 et 3 )

Tandis que le $5^{\mathrm{e}}$ est formulé comme suit :

5. Mieux comprendre les perceptions de la femme atteinte de cancer du sein à l'égard du dépistage de la détresse (phase 2)

\section{Considérations éthiques}

Nous reprenons ici les considérations éthiques déjà décrites dans la partie 1 de l'article. Cette étude a obtenu, en début d'année 2013, un certificat éthique de l'établissement où s'est déroulée l'étude. Il est important de noter que le dépistage systématique de la détresse étant considérée comme une intervention standard en oncologie, il n'y a pas de consentement autre que l'accord verbal de la clientèle au moment du dépistage. Toutefois, un consentement écrit a été obtenu de la part des participants pour toutes les autres phases de l'étude y compris la phase 2 qui fait l'objet de cet article.

\section{Collecte de données et analyses}

Les outils utilisés pour cette phase de l'étude, soit les questionnaires de perceptions des infirmières (QPI) et de celles de la clientèle (QPC) ont été élaborés à partir notamment de résultats d'étude (Asselin, 2012; Blais, 2012) concernant le dépistage systématique de la détresse présentés en novembre 2012 au CHU de Québec lors d'une journée scientifique en oncologie. De plus, deux questionnaires élaborés en langue anglaise par le Alberta Health Services (AHS, 2012 A et $\mathrm{B}$ ) pour connaitre la perception de la clientèle concernant la qualité des soins et aussi le point de vue du clinicien sur le sujet de l'outil de dépistage de la détresse dans le cadre du projet EDOPI/IPODE pour l'implantation du dépistage de la détresse ont été utilisés. ${ }^{1}$ Ces questionnaires ont également été bonifiés suite aux résultats de focus group réalisés auprès d'infirmières dans le cadre de l'implantation du dépistage de la détresse à notre centre. Les outils de l'AHS ont été traduits et adaptés avec permission.
Le QPC est un questionnaire mixte (quantitatif et qualitatif) comprenant 15 questions avec des sous-questions. Il porte sur divers aspects liés au dépistage de la détresse notamment la facilité de la complétion de l'ODD, son utilité, si le dépistage peut être émotionnellement dérangeant, l'aide demandée et l'aide reçue.

Le QPI se compose de 15 questions avec des sous-questions. Il s'agit également d'un questionnaire mixte. Il aborde notamment le sens accordé au dépistage par l'infirmière, l'importance accordée par elle et l'importance qu'elle perçoit de la part du patient ainsi que le degré de confort qu'elle a en lien avec divers aspects de la pratique infirmière autour du dépistage.

Des tests paramétriques ont été réalisés lorsque la taille de l'échantillon le permettait. L'analyse qualitative a été réalisée selon une approche simple de catégorisation par les 2 chercheures principales de l'étude (NT et OR) de manière indépendante dans un premier temps. Elles ont laissé émerger les thèmes après plusieurs lectures des réponses manuscrites obtenues. Ensuite, une première catégorisation a été créée pour chacune des questions, puis une réduction des thèmes a été opérée par les chercheures. Ensuite elles ont comparé les catégories ainsi obtenues. Pour l'ensemble des questions, un consensus quasi parfait a été obtenu dès la première comparaison. Des discussions ont permis ultérieurement le consensus pour les catégories restantes.

\section{RÉSULTATS}

\section{Caractéristiques des participants \\ Participants clientèle}

Léchantillon de convenance comprend 122 personnes atteintes de cancer, soit 80 participantes cancer du sein et 42 patients cancers hématologiques. Le recrutement s'est déroulé au cours de l'année 2014. Pour participer à l'étude, tous les participants devaient avoir complété au moins une fois l'ODD. Ceux qui n'avaient pas complété l'ODD ont été exclus de l'étude. Aucune donnée sociodémographique n’a été collectée à l'aide de ce questionnaire. Toutefois, pour la deuxième phase de l'étude, ce sont également les mêmes groupes de participants qui avaient été sollicités lors de la première phase. Phase pour laquelle nous avons des informations sur l'âge et le diagnostic. Il est donc raisonnable de penser que les informations recueillies en ce qui concerne l'âge et le diagnostic peuvent être transposées.

\section{Participants infirmières}

Léchantillon de convenance comprend 45 infirmières, dont la majorité $(64,4 \%)$ exerce auprès de la clientèle de cancer hématologique. Une plus petite proportion exerce principalement auprès de la clientèle de cancer du sein (13,3\%) et $20 \%$ auprès de diverses clientèles d'oncologie (auprès de personnes

1. EDOPI/IPODE. Un programme éducatif de dépistage de la détresse en ligne a été conçu pour former le personnel (principalement les infirmières). Expérience globale du cancer (2012) PCCC. Dépistage de la détresse, le Ge signe vital : guide d'adoption de pratiques exemplaires pour des soins centrés sur la personne. www.vuesurlecancer.ca. 
sous traitement de chimiothérapie ou de radiothérapie pour divers cancers). Cette distribution des participants infirmières correspond à la distribution réelle des infirmières qui œuvrent auprès des diverses clientèles au sein du programme de cancérologie de notre établissement. Ainsi elles sont plus nombreuses à exercer auprès d'une population atteinte de cancers hématologiques car elles sont rattachées à l'unité d'hospitalisation qui couvre 3 quarts de travail comparativement aux infirmières qui œuvrent en ambulatoire, tous types de cancers confondus dont celles qui exercent uniquement auprès de femmes atteintes de cancer du sein.

\section{Perceptions des patients}

Présentation de l'outil

Sur les 122 patients participants, la grande majorité s'est vue présenter l'outil de dépistage, soit $97,5 \%$ des patients. Ce sont 3 patientes atteintes de cancer du sein qui rapportent que l'outil ne leur a pas été présenté.

\section{Facilité de complétude}

La majorité des participants à l'étude (77\%) trouvent l'outil de dépistage facile à compléter. Un certain nombre de participants $(17,2 \%)$, trouve plus ou moins facile la complétion de l'outil voire même difficile à compléter (3,3\%). L'information était manquante pour 3 personnes. Plus de patients atteints de cancer hématologique 7,5\% trouvent l'outil difficile à remplir contre $1,3 \%$ cancer du sein, cette différence n'est pas significative.

\section{Ce questionnaire sert à}

À la question "Selon vous à quoi sert ce questionnaire? » deux thèmes centraux se dégagent de l'ensemble des réponses à cette question de la part des participants. Le premier thème regroupe les verbatim exprimant l'idée de l'évaluation ou du dépistage de leur détresse, de leur stress, de leur anxiété, de leurs besoins ou encore de leurs préoccupations.

À noter que le $\mathrm{H}$ précédent le numéro du participant indique qu'il s'agit d'un participant atteint de cancer hématologique et le $\mathrm{S}$ indique un participant atteint de cancer du sein.

Le verbatim suivant illustre ce thème de l'évaluation ou dépistage de la détresse ou des besoins :

S39 « Il sert à dépister la détresse psychologique face au vécu

du cancer du sein (dans mon cas). »

L'autre thème qui ressort des verbatim en réponse à la question : à quoi sert ce questionnaire touche à la dimension d'aide. Les participants ont ainsi répondu que l'ODD sert à les aider ou à aider les professionnels. L'outil sert ainsi à voir si la personne a un besoin d'aide ou à identifier les actions nécessaires à poser pour l'aider ou à les diriger vers des ressources. Les deux verbatim suivants illustrent ce thème :

$S 2$ «À mesurer le degré d'aide que les personnes atteintes ont besoin et quelle sorte d'aide leur apporter. »

H14 «Sert à valider mes craintes et mesurer mon niveau d'anxiété afin de m'orienter vers les ressources nécessaires, si besoin est. »
Utilité perçue et raisons évoquées pour expliquer la réponse

À la question : avez-vous trouvé utile de compléter le questionnaire de dépistage de la détresse? La très grande majorité des participants, ont répondu oui, soit plus de $81 \%(81,1)$. Quatre personnes ont répondu à la fois oui et à la fois non. Leurs réponses ont été versées dans la catégorie « oui » suite à la lecture des réponses obtenues lorsqu'on leur demandait de préciser les raisons derrière leurs choix de oui ou non. La figure 1 présente les résultats à cette question.

Le thème majeur qui ressort de l'analyse des raisons indiquées par les tenants du oui fait référence au caractère introspectif de l'outil de dépistage. À notre connaissance aucune étude n'avait mis en perspective cet élément. Selon nos participants, la complétion de l'outil permet un temps servant à faire le point sur ce qui est ressenti ou vécu à ce moment-là, comme en témoignent les verbatim suivants.

$S 2$ «À ce moment je me sentais plus forte. Mais en lisant les questions, je me suis sentie plus faible que je l'aurais cru. Et cela m'a surprise. »

S69 «Oui, c'est à ce moment-là que j'ai compris que je vivais beaucoup de colère face à ce diagnostic et que j'ai ressenti un profond sentiment d'injustice »

S76 « Cela m’a permis de clarifier mon état de stress et de voir quels sont mes besoins ».

D'autre part, l'outil est également perçu utile par les participants patients parce qu'il permet une forme de partage ou de communication autour de l'expérience ou des émotions.

S38 «Oui, j'ai pu partager certaines angoisses que je n'avais pas partagées avec ma famille même minimes soient-elles. Cela m'a soulagé. »

L'outil permet également d'être rassuré sur ce qui peut être fait ou proposé comme aide si le besoin se fait sentir.

S18 «Oui, car après l'annonce d'un cancer on se sent dépas-

sée par les événements. Il est bien de savoir que l’on peut obte-

nir de l'aide... »

Par ailleurs, 15 participants ont justifié leur non par l'absence de détresse ou par l'absence d'un ressenti d'un besoin d'aide comme en témoigne les verbatim des participants $\mathrm{H} 5$ et H30, S23 et S 67.

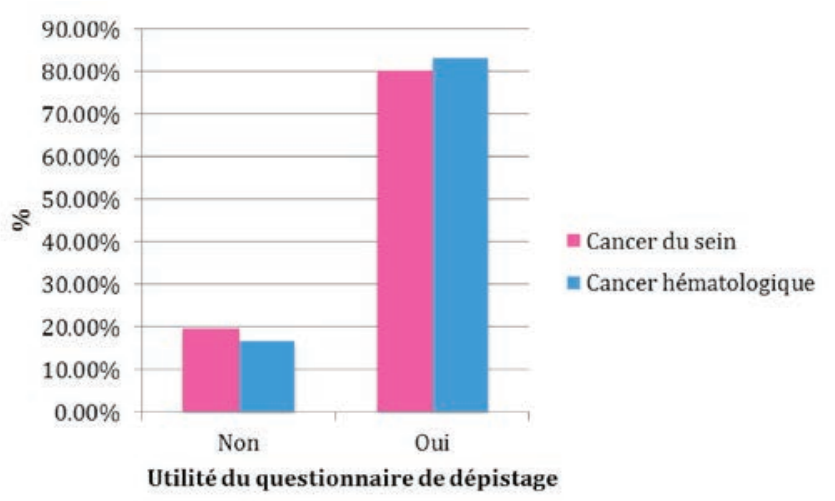

Figure 1. Utilité du questionnaire de dépistage 
H5 « Je dis non à cause que je savais déjà à quoi m'attendre avec l'hospitalisation donc je n'ai pas eu de détresse lors de mon séjour ».

H30 «Je n'étais pas en détresse ».

S23 «Ne ressentant pas d'angoisse ou d'autre symptôme, cela n'a pas été utile sauf peut-être de réaliser que je vivais la situation assez bien. »

S67 « Parce que je n'avais pas vraiment besoin d'aide. »

Par contre parmi les non, une participante a signalé qu'on n'avait pas fait de suivi auprès d'elle et une autre a rapporté que rien n'avait été fait avec les réponses données par elle.

\section{Inconfort émotionnel ou gêne à compléter l'outil et raisons évoquées}

À nouveau une très grande majorité de patients, 89,3\% d'entre-deux (soit 109 patients) déclarent ne pas avoir trouvé le questionnaire de dépistage de la détresse dérangeant ou incommodant. À l'inverse, $9 \%$ des patients (soit 11 patients) ont trouvé le questionnaire de dépistage dérangeant ou incommodant. Enfin, deux patients ne se sont pas prononcés sur cette question. À noter que parmi les 11 personnes qui ont répondu oui. Cinq ont répondu oui parce qu'en effet la complétion du questionnaire a fait surgir des émotions ou a permis une prise de conscience de ce qui est réellement vécu comme en témoignent les participants H3, S69 et S70.

H3 « Ça fait monter les émotions. C'est un mal nécessaire. »

S69 «Même s'il est utile. Sur le coup il est dérangeant, car il m'a obligée à m'interroger sur ce que je vivais. Honnêtement, je crois que je refusais encore d'admettre que j'avais reçu un diagnostic de cancer du sein. »

S70 «En constatant que le vécu du cancer du sein peut m'affecter autant, ainsi que mes proches. »

\section{Aide et services proposés}

À la question : Après avoir complété le questionnaire de dépistage de la détresse, est-ce qu'un professionnel a discuté avec vous de vos réponses? Plus de $40 \%$ ont répondu non (40,2\%). La figure 2 fait part des résultats à cette question par type de cancer.

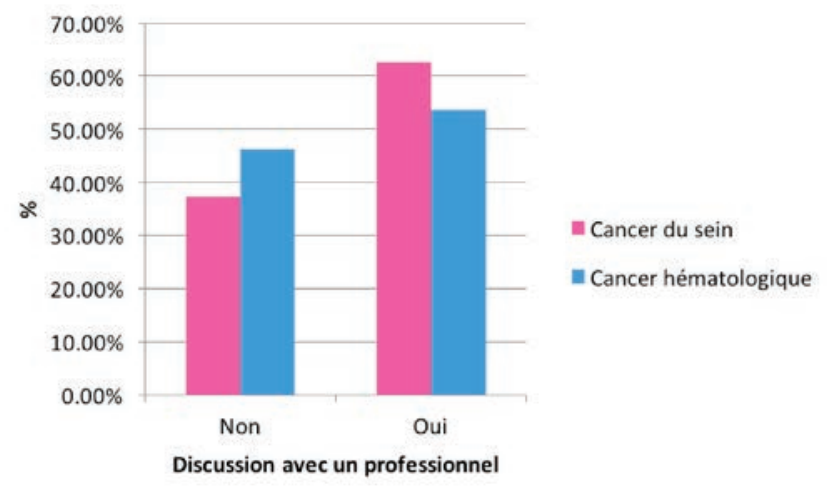

Figure 2. Discussion avec un professionnel post-complétion de l'outil?
À la question : vous a-t-on proposé l'aide qu'il vous fallait pour répondre aux besoins et aux préoccupations que vous avez exprimés, $71,4 \%$ des patientes atteintes de cancer du sein et $66,7 \%$ des patients atteints de cancer hématologique se sont vus proposer l'aide qu'il leur fallait pour répondre aux besoins et aux préoccupations qu'ils avaient exprimés. Il reste donc $28,4 \%$ des patientes atteintes de cancer du sein et 33,3\% des patients atteints de cancer hématologique qui ne sont pas vus proposer l'aide qu'il leur fallait. Majoritairement encore une fois les motifs des non s'expliquent en grande partie parce que ces personnes n'ont pas exprimé un besoin d'aide comme en témoignent les verbatim des deux participants suivants.

H2 « Non, j'ai toujours mentionné que je n'avais pas besoin d'aide particulière. »

S2 «Si j'avais besoin d'aide, j'étais pour la rappeler sans faute».

Par contre, d'autres personnes n'ont pas reçu l'aide espérée en réponse à leurs besoins.

S9 «Personne ne m'a rappelé à ce sujet. Il y a ma famille qui m'entoure, c'est déjà gros. »

S75 «On m'a surtout référée à la psychologue, mais mon besoin aurait été davantage d'être soutenue par le milieu médical (infirmière et médecin). »

À la question : si oui avez-vous accepté cette aide? 62,3\% des patientes ayant un cancer du sein ont accepté alors que $60,9 \%$ ayant un cancer hématologique ont accepté l'aide offerte et 36,7 \% l'ont refusé. Voir la figure 3.

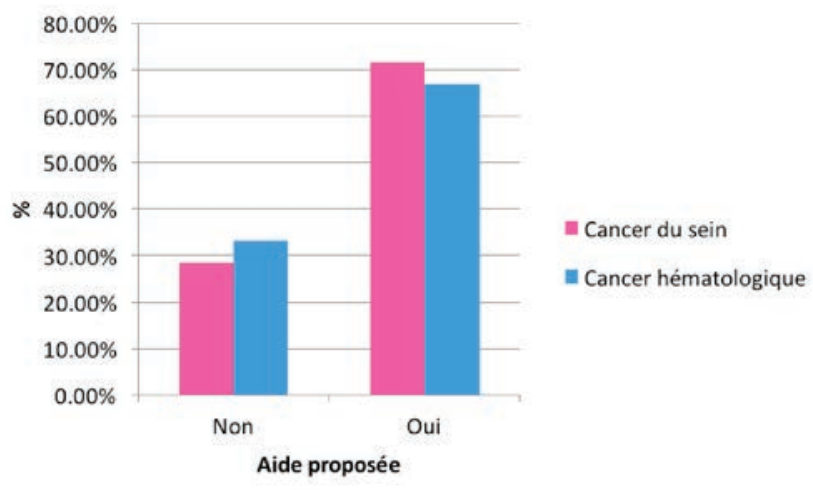

Figure 3. Aide proposée perçue comme adéquate en réponse aux besoins exprimés

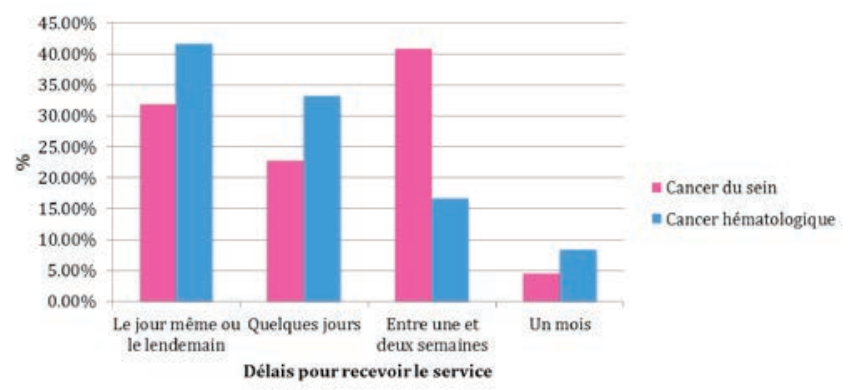

Figure 4. Délais pour recevoir le service 
Voici un aperçu des précisions apportées à cette question par les participants :

S33 « Non. Mais je n'ai pas vraiment besoin pour le moment du moins. Je laisse donc la place aux plus démunis. »

S67 « Non J'avais assez de force et ma famille est autour de moi pour m'aider à traverser cette épreuve. »

S12 «Oui, je crois qu'il faut encourager le personnel médical à proposer de l'aide, que ça devienne comme une habitude, un réflexe. "

S69 «Oui. La première fois j'ai refusé, mais lorsque j'ai appris que je devais aussi recevoir des traitements de chimio, mon IPO m'a à nouveau suggéré de rencontrer la psychologue et j'ai accepté. »

Services reçus et délais.

Sur les 47 patients qui ont accepté l'aide proposée, 34 ont reçu un service et 13 patients n'ont pas reçu le service proposé, soit 27,7 \%. La figure 4 indique les délais survenus avant de recevoir le service. Plus de $60 \%$ des participants reçoivent le service à l'intérieur de quelques jours, et pour 35,3\% d'entre eux, le jour même ou le lendemain.

\section{Niveau de satisfaction global (tableau 1)}

Au total, seulement $1,6 \%$ et 3,3\% des patients (soit 6 patients au total) déclarent respectivement ne pas être du tout satisfait ou être peu satisfait par l'identification de leur détresse et de l'aide offerte jusqu'ici. À l'inverse, la grande

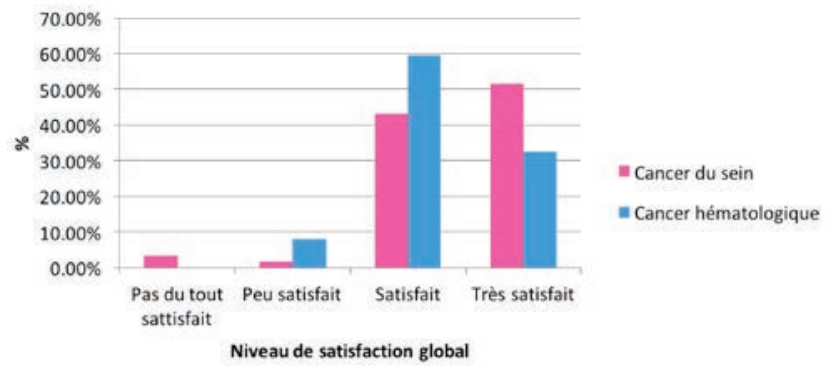

Figure 5. Niveau de satisfaction global selon type de cancer majorité des patients déclarent être satisfaits (38,5\%) et très satisfaits (36,1\%). Cette proportion augmente considérablement si l'on ne prend en compte que ceux ayant précisé un choix de réponse. À noter ici que 20,5\% des patients n'ont pas répondu à cette question. Voir la figure 5 pour le niveau de satisfaction global. Le niveau de satisfaction global des deux groupes de patients est différent au seuil de $1 \%$. (Khi2 =7 236; p. $=0,065)$.

\section{Échelle d'évaluation des symptômes d'Edmonton (ESAS)}

Près des trois quarts des patients $(71,35)$ sont entièrement d'accord avec l'énoncé suivant : L'ESAS est important parce qu'il aide mon équipe de soins à savoir quels sont mes symptômes et leur gravité. Tout de même plus d'un patient sur 10 (13,1 \%) est plus ou moins d'accord avec l'énoncé. Le tableau 2 donne des précisions en ce qui concerne la distribution des réponses des participants.

Une moins grande proportion de patients $(57,4 \%)$ est d'accord avec l'énoncé suivant à savoir que l'équipe de soins tient compte de la notation ESAS quand elle détermine le traitement. Il faut dire que près de $14 \%(13,9 \%)$ de l'échantillon n'a pas répondu à cette question. Et la même proportion était sans opinion à ce sujet.

La figure 6 donne, en fonction du type de cancer, le pourcentage d'accord avec l'énoncé suivant : mes douleurs et mes symptômes ont été réduits à un niveau où je suis confortable. Retenons que $23 \%$ de l'échantillon ont répondu toujours et près de $42 \%(41,8)$ la plupart du temps.

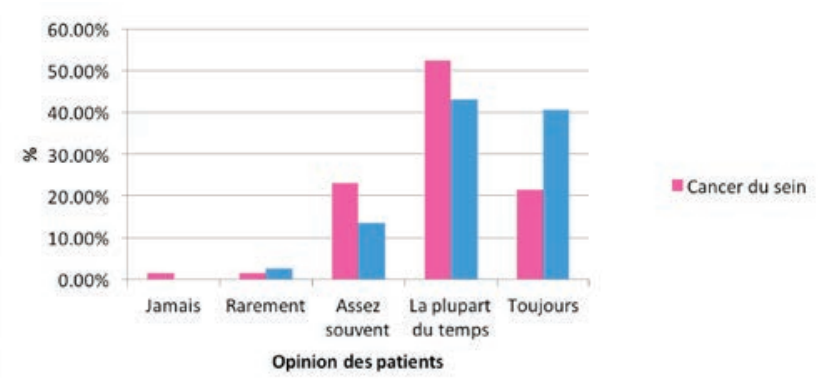

Figure 6. Mes douleurs et mes symptômes ont été réduits à un niveau ou je suis confortable

Tableau 1. Quel est votre niveau de satisfaction global en ce qui a trait à l'identification de votre détresse et l'aide offerte jusqu'ici

\begin{tabular}{|l|l|l|l|l|}
\hline & Fréquence & Pourcentage & Pourcentage valide & Pourcentage cumulé \\
\hline Valide & & & & \\
\hline Pas du tout satisfait & 2 & 1,6 & 2,1 & 2,1 \\
\hline Peu satisfait & 4 & 3,3 & 4,1 & 6,2 \\
\hline Satisfait & 47 & 38,5 & 48,5 & 54,6 \\
\hline Très satisfait & 44 & 36,1 & 45,4 & 100,0 \\
\hline Total & 97 & 79,8 & 100,0 & \\
\hline Manquant & 25 & 20,5 & & \\
\hline Total & 122 & 100,0 & & \\
\hline
\end{tabular}


Tableau 2. L'ESAS est important parce qu'il aide mon équipe de soins à savoir quels sont mes symptômes et leur gravité

\begin{tabular}{|l|l|l|l|l|}
\hline & Fréquence & Pourcentage & Pourcentage valide & Pourcentage cumulé \\
\hline Valide & & & & \\
\hline Entièrement en désaccord & 1 & 0,8 & 0,9 & 0,9 \\
\hline Plus ou moins en désaccord & 3 & 2,5 & 2,6 & 3,4 \\
\hline Plus ou moins d'accord & 16 & 13,1 & 13,8 & 17,2 \\
\hline Entièrement d'accord & 87 & 71,3 & 75 & 92,2 \\
\hline Sans opinion & 9 & 7,4 & 7,8 & 100,0 \\
\hline Total & 116 & 95,1 & 100,0 & \\
\hline Manquant & 6 & 4,9 & 100,0 & \\
\hline Total & 122 & & & \\
\hline
\end{tabular}

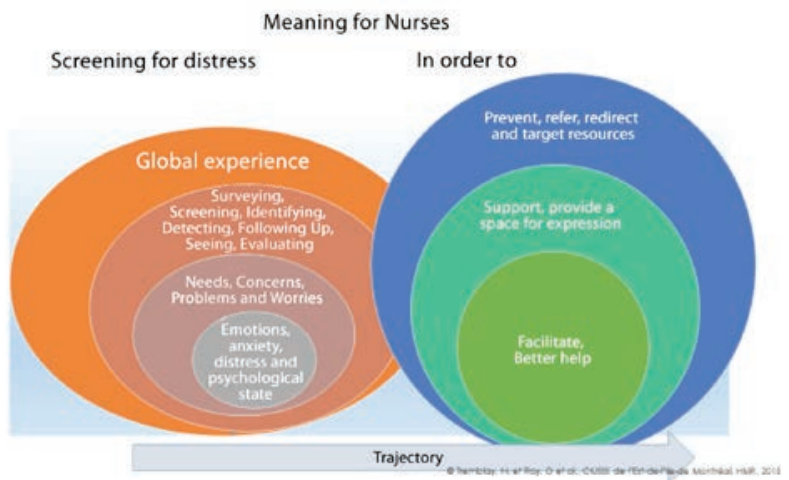

Figure 7. Signification du dépistage pour les infirmières

Perceptions des infirmières

Signification du dépistage de la détresse

À la question : dépister la détresse, ça signifie quoi pour vous comme infirmière? Deux grandes catégories de thèmes ont émergé. La première regroupe divers verbes utilisés pour décrire l'identification, lévaluation, le dépistage, le repérage des problèmes, des émotions, des préoccupations de la clientèle à travers une trajectoire de soins qui s'inscrit dans une expérience plus globale. La deuxième catégorie comprend les divers verbes utilisés pour indiquer le but, la finalité de la démarche : mieux aider, soutenir, diriger vers des ressources. La figure 7 renvoie au modèle théorique émergent de la signification du dépistage de la détresse pour les infirmières élaboré par les chercheurs Tremblay et Roy à partir des thèmes utilisés par les infirmières.

\section{L'importance du dépistage selon les infirmières}

À la question suivante : «Jusqu'à quel point pensez-vous qu'il est important de dépister systématiquement la détresse des patients? », la majorité des infirmières ont répondu « important ». En effet, 60 \% des infirmières considèrent qu'il est important de dépister systématiquement la détresse des patients, 33,3\% des infirmières considèrent qu'il est incontournable de dépister systématiquement la détresse et enfin, $6,7 \%$ considèrent que c'est moyennement important. Il est intéressant de constater qu'aucune infirmière ne considère que cela n'est pas du tout important ou peu important.
Perception des infirmières des perceptions des patients

Selon les participantes infirmières, les patients ne perçoivent pas la même importance en ce qui concerne le dépistage systématique de la détresse qu'elles-mêmes y voient (22,7 \% des infirmières). Seuls $11,1 \%$ des infirmières considèrent que les patients perçoivent la même importance concernant le dépistage de la détresse. Une grande proportion des infirmières $(64,4 \%)$ ont répondu « autre » à cette question. Et l'analyse qualitative de ces « autre » indique qu'elles ont presque tout écrit : « Ça dépend, pour certains oui, pour d'autres non ». À partir des commentaires des participantes, on peut déduire que leurs perceptions découlent de nombreux facteurs survenant, soit dans l'interaction, soit tributaires du contexte tels que : le moment du dépistage, le degré d'attention ou la vitesse avec lequel le patient répond, s'il donne l'impression que cela l'intéresse ou non, s'il perçoit avoir besoin d'aide ou non, ou s'il refuse ou non les références offertes. Bref leurs diverses expériences de dépistage influencent ces perceptions.

I8 «Je crois que cela dépend de la façon dont l'ODD est expliqué et présenté au patient ainsi qu’à létat du patient à l'arrivée. »

I15 «C'est très variable d'un patient à l'autre. Certains trouvent l'ODD important et d'autres semblent ennupés lorsqu'on leur présente. »

I26 «Certains peut-être que oui (ceux qui acceptent l'aide plus facilement), mais pour d'autres c'est de la paperasse de plus à remplir au moment où ils ont déjà beaucoup de choses à gérer ou ils sont en train d'essayer de comprendre ce qui se passe ».

I29. «Ça dépend des patients. Je crois que même si on leur explique d'être sincères et que c'est pour eux qu'ils le remplissent, certains veulent juste s'en débarrasser ou sont trop orgueilleux pour demander de l'aide, mais c'est loin d'être la majorité.

Le non des infirmières à cette question s'appuie aussi sur ce qui est perçu de leur part venant du patient et qui n'est pas nécessairement validé.

I4 « Non, beaucoup croient qu'aucune aide n'est possible. »

I17 « Non, j'ai l'impression qu'ils se disent que ce n'est pas grave, que ça va passer.» 
Tableau 3. Perception de l'utilité du thermomètre de détresse et de la liste de problèmes

\begin{tabular}{|l|l|l|l|l|}
\hline & Fréquence & Pourcentage & Pourcentage valide & Pourcentage cumulé \\
\hline Valide & & & & \\
\hline Pas du tout utile & 6 & 13,3 & 13,3 & 13,3 \\
\hline Assez utile & 26 & 57,8 & 57,8 & 71,1 \\
\hline Très utile & 13 & 28,9 & 28,9 & 100,0 \\
\hline Total & 45 & 100,0 & 100,0 & \\
\hline
\end{tabular}

Tableau 4. Perception de l'utilité de l'ESAS

\begin{tabular}{|l|l|l|l|l|}
\hline & Fréquence & Pourcentage & Pourcentage valide & Pourcentage cumulé \\
\hline Valide & & & & \\
\hline Pas du tout utile & 1 & 2,2 & 2,2 & 2,2 \\
\hline Assez utile & 24 & 53,3 & 53,3 & 55,6 \\
\hline Très utile & 20 & 44,4 & 44,4 & 100,0 \\
\hline Total & 45 & 100,0 & 100,0 & \\
\hline
\end{tabular}

I12. « Moyennement, certains sont agacés de devoir remplir ce document qui les force peut-être à mettre des mots sur leurs difficultés, par pudeur ou parce qu'ils ne sont pas prêts. »

Utilité du thermomètre, de la liste de problèmes et de l'ESAS

La majorité des infirmières (57,8 \%) juge assez utile le thermomètre et la liste de problèmes qui l'accompagne. Elles sont $28,9 \%$ à trouver qu'il est très utile et $13,3 \%$ à la trouver pas du tout utile. En comparaison, elles sont beaucoup moins nombreuses à trouver l'échelle d'évaluation des symptômes pas $\mathrm{du}$ tout utile $(2,2 \%)$. Les infirmières considèrent majoritairement que l'ESAS est assez utile (53,3\%) et elles sont assez nombreuses à considérer que l'ESAS est très utile (44,4\%). Les tableaux 3 et 4 présentent ces résultats.

\section{Influences positives et négatives du dépistage sur leur} pratique infirmière

Il était demandé aux infirmières d'identifier, parmi une liste proposée d'énoncés, de quelle façon le dépistage de la détresse influence positivement et négativement leur pratique. Les énoncés positifs sont les suivants; permet une évaluation plus pointue, permet plus de contacts avec les personnes qui ont besoin d'une gestion de symptômes, permet une compréhension plus élargie de ce qui se passe avec la personne, permet de mieux documenter, et autre aspect). La liste des énoncés négatifs comprend : prend du temps, collecte des données non pertinentes, rend les personnes trop émotives, me rend inconfortable comme clinicien, et autre aspect.). La proportion des infirmières qui trouve que cela prend du temps est plus grande pour celles qui exercent en ambulatoire comparativement à celles qui exercent auprès d'une clientèle hospitalisée. Les figures 8 et 9 présentent les items cochés par les infirmières et dans quelle proportion.

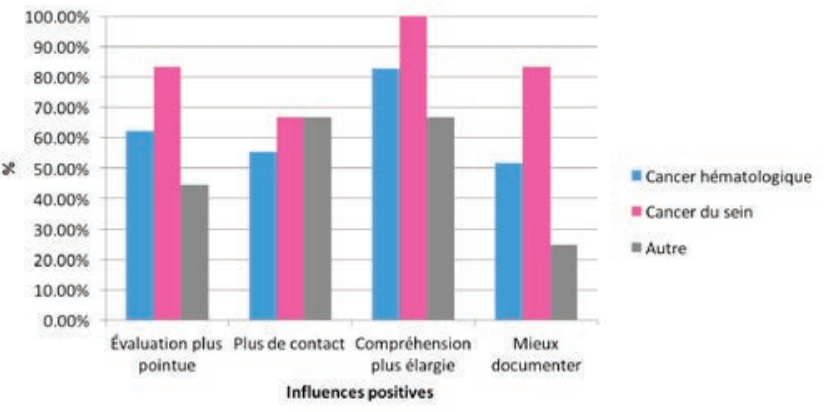

Figure 8. Influences positives du dépistage

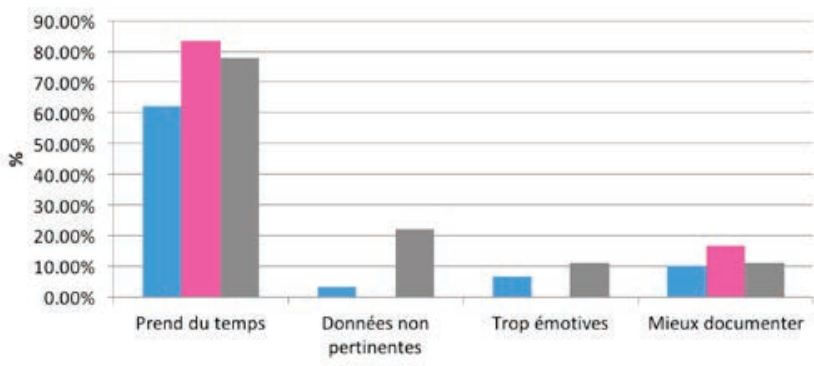

Figure 9. Influences négatives du dépistage sur la pratique

\section{Références offertes}

Suite au dépistage de la détresse, 86,7 \% des infirmières proposent aux patients une rencontre avec un psychologue. Il s'agit de la référence la plus fréquemment offerte. Ensuite, $71,1 \%$ des infirmières proposent une rencontre avec un travailleur social. Moins de la moitié des infirmières offre comme référence un nutritionniste $(42,2 \%)$, un conseiller clinicien (33\%), un médecin (26,7\%) et un physiothérapeute (13\%). 
Enfin, près d'un tiers des infirmières propose une autre référence $(31,1 \%)$. Il s'agit alors d'une référence vers un intervenant en soins spirituels, un psychiatre, un bénévole ou un groupe communautaire.

\section{Sentiment de confiance face aux étapes du dépistage et aux} domaines de problèmes ou de préoccupations

Les réponses obtenues par les participantes suggèrent que les infirmières se sentent plus à l'aise à identifier et évaluer la détresse qu'à y répondre et fournir un soutien structuré. Ainsi elles sont plus nombreuses à se dire confiante ou très confiante dans le dépistage $(81,1)$ et l'évaluation $(55,6 \%)$ alors que moins de la moitié se disent confiantes ou très confiantes pour répondre à la détresse $(46,7 \%)$ ou fournir un soutien structuré $(36,4, \%)$ et $13,6 \%$ ne se sentent pas du tout confiantes à cet égard.

Il est intéressant de noter que les résultats montrent que c'est dans les domaines des besoins informationnels $(75,5 \%)$ et physiques $(84,5 \%)$ que les infirmières se sentent confiantes ou très confiantes. Parmi ces sept catégories de besoins, les résultats des tests de khi2 montrent que le sentiment de confiance des infirmières varie de manière significative selon leurs patientèles pour deux catégories de besoins : les besoins émotionnels $\left(\mathrm{khi}^{2}=17,07 ; \mathrm{p} .=0,009\right)$ et les besoins psychosociaux $\left(\mathrm{khi}^{2}=15,82 ; \mathrm{p} .=0,045\right)$.

Par ailleurs, pour les besoins émotionnels, les infirmières lorsqu'elles travaillent auprès de patientes atteintes de cancer du sein semblent plus en confiance. En effet, 33,3\% des infirmières travaillant auprès de patientes atteintes de cancer du sein se sentent très en confiance pour répondre aux besoins émotionnels des patients, contre $0 \%$ des infirmières en cancers hématologiques et $0 \%$ des infirmières travaillant auprès d'une autre patientèle. Cette tendance est la même pour les besoins psychosociaux. En effet 6,9 \% des infirmières exerçant auprès de la clientèle hémato-greffe ne se sentent pas du tout confiantes, et $0 \%$ d'entre elles se sentent très confiantes. Elles se sentent assez confiantes $(20,7 \%)$, modérément confiantes $37,9 \%$. À peine $34 \%$ d'entre elles se sentent confiantes.
Le tableau 5 et la figure 10 présentent les résultats ayant trait à la question : "Jusqu'à quel point vous sentez-vous confiante face aux divers domaines de besoins? »

\section{Expérience des infirmières avec le dépistage}

Soixante-deux pour cent des participantes infirmières avaient dépisté à plus d'une dizaine de reprises la clientèle. Deux infirmières avaient une centaine de dépistages à leur actif, 9 une vingtaine de fois et plus. Parmi les autres, une réponse était manquante, 15 infirmières (soit $33 \%$ ) avaient 5 dépistages et moins à leur actif. Autour de $5 \%$ se situaient entre 5 et 10 dépistages. On a demandé également de se prononcer combien de ces expériences ont été plus difficiles et combien ont été plus positives. Pour 57,8\% de l'échantillon au moins une expérience a été plus difficile. Trois infirmières n'ont pas répondu à cette question. Parmi celles qui ont beaucoup dépisté, une seule infirmière en a vécu plusieurs de difficiles, soit 25 sur une centaine. Une infirmière a répondu que sur les 2 fois où elle a dépisté, toutes ses expériences ont été difficiles.

I4. Je ne me sens pas outillée.

Pour $44,4 \%$ des infirmières, il y a eu plus d'expériences plus positives que difficiles reliées à l'outil de dépistage, $13 \%$ ont exprimé qu'elles ont vécu autant d'expériences positives que négatives. Deux infirmières ne se sont pas prononcées. Deux autres ne pouvaient répondre. Toutefois, $15,6 \%$ des infirmières n'ont vécu aucune expérience plus positive.

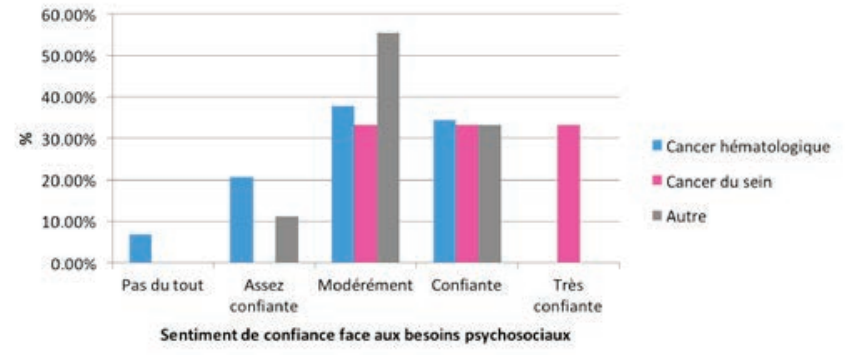

Figure 10. Sentiments de confiance face aux besoins psychosociaux

Tableau 5. Sentiment de confiance face aux besoins

\begin{tabular}{|l|l|l|l|l|l|l|l|}
\hline & informationnels & physiques & émotionnels & psychosociaux & $\begin{array}{l}\text { résistance } \\
\text { à accepter } \\
\text { l'orientation } \\
\text { vers un } \\
\text { intervenant }\end{array}$ & $\begin{array}{l}\text { des groupes } \\
\text { minoritaires }\end{array}$ & $\begin{array}{l}\text { face aux } \\
\text { inquiétudes } \\
\text { relatives au } \\
\text { deuil, mort }\end{array}$ \\
\hline Pas du tout & 4,4 & 0 & 0 & 4,4 & 4,8 & 16,7 & 4,4 \\
\hline Assez confiante & 4,4 & 4,4 & 15,9 & 15,6 & 16,7 & 14,3 & 22,2 \\
\hline Modérément & 15,6 & 11,1 & 29,5 & 40 & 19 & 33,3 & 28,9 \\
\hline Confiante & 53,3 & 66,7 & 47,7 & 33,3 & 50 & 35,7 & 40 \\
\hline Très confiante & 22,2 & 17,8 & 6,8 & 6,7 & 9,5 & 0 & 4,4 \\
\hline Total & 100,0 & 100,0 & 100,0 & 100,0 & 100 & 100 & 100 \\
\hline
\end{tabular}




\section{Aspects qui fonctionnent bien ou moins bien par rapport au dépistage}

On note, parmi les thèmes les plus fréquemment relevés par les infirmières comme étant des aspects associés au dépistage qui fonctionnent bien, ceux-ci : le dépistage permet de dépister plus rapidement et au moment opportun les problèmes et d'offrir les services appropriés, il permet aussi une plus grande ouverture sur les besoins des patients, un meilleur dialogue, il permet une meilleure évaluation, un meilleur suivi. Par contre elles sont nombreuses à avoir mentionné le manque de temps pour approfondir les réponses obtenues, le manque d'intimité avec le patient et le temps choisi pour le dépistage qui ne serait pas favorable, donnant comme exemple le dépistage à l'admission où le lien avec le patient peut ne pas s'être encore créé.

\section{DISCUSSION}

Bien qu'il y ait convergence des perceptions des infirmières et des patients sur l'importance ou l'utilité du dépistage de la détresse, il existe également une divergence à ce propos. La quasi-totalité des infirmières $(93,3 \%)$ juge important voire incontournable le dépistage de la détresse et la grande majorité des patients $(81,1)$ trouve utile ce dépistage. Toutefois, l'étude a démontré qu'à peine plus d'une infirmière sur 10 (11,1\%) perçoit que le patient accorde la même importance qu'elles au dépistage. Elles ont répondu majoritairement (64,4\%) : ça dépend. Et l'analyse des perceptions infirmières révèle que divers comportements des patients et leurs interprétations nourrissent cette perception. Ainsi les refus de compléter le questionnaire, le caractère expéditif de certains à le compléter, le temps écoulé avant qu'il soit complété lorsque remis à l'admission sont perçus comme des signes que le dépistage n'est pas si important pour les patients.

Par ailleurs, nous avons rapporté dans la partie 1 de cet article qu'une vaste majorité de patients coche qu'ils ne souhaitent pas être aidés pour des problèmes ou des préoccupations mentionnées sur l'ODD : 77,3\% au temps 1 et 85,3\% au temps 2. On se rappelle que le temps 1 correspond pour les patients atteints de cancers hématologiques à l'admission à l'unité hospitalisation et que le temps 2 correspond le plus souvent au congé. Alors que pour les femmes atteintes de cancer du sein, le temps 1 correspond à une $2^{\mathrm{e}}$ ou $3^{\mathrm{e}}$ rencontre suivant l'annonce du diagnostic et le temps 2 correspond à un jour $\mathrm{X}$ dans la période des traitements.

De plus, les participants à l'étude sont nombreux à refuser les références proposées. Parmi les $46 \%$ de l'échantillon se voyant offrir une référence au temps 1 , celle-ci est acceptée dans $40 \%$ des cas. Sans compter qu'une certaine proportion de patients refusent de compléter le questionnaire parce qu'ils n'en ressentent pas le besoin (raison principale des refus de compléter l'outil en phase 1). De plus, l'étude a démontré que le motif le plus souvent indiqué par le patient lorsqu'il perçoit l'ODD comme étant non utile est qu'il n'en ressent pas le besoin.

Quel est l'effet de ces perceptions sur le transfert et l'appropriation de cette pratique par les infirmières? Difficile de répondre. Si certaines infirmières ont écrit dans la section de ce qui fonctionne moins bien avec le dépistage : les refus, la réticence, la résistance de certains patients surtout des hommes de parler de leur douleur psychologique et le besoin d'être mieux outillée pour agir dans ces situations, beaucoup reste encore à découvrir. Cet aspect mérite définitivement d'être davantage approfondi par la recherche et la clinique tout comme il doit être l'occasion d'échanges lors de la formation des intervenants sur le dépistage. Déjà en 2008, Steele et Fitch (2008) mentionnaient qu'il peut être difficile pour les professionnels de reconnaître l'autonomie des personnes atteintes de cancer et que ces dernières peuvent décliner l'aide offerte, car celle-ci peut ne pas être désirée. Certaines infirmières ont aussi mentionné l'absence d'expériences plus positives avec le dépistage. À quoi l'attribuer? Préparation et formation inadéquate? Inconfort, embarras, croyances contraignantes à l'égard du dépistage? Collaboration déficiente? Encore là, plus de questions que de réponses. Certains commentaires de nos participantes infirmières pointent vers un besoin de formation pour développer des habiletés plus avancées en matière de soutien.

On peut en arriver à douter également de l'utilité du dépistage si le patient exprime ne pas en avoir besoin. Mais de quel besoin nous parle-t-il ici? S'agit-il d'un besoin d'aide ou plutôt de l'absence de ce besoin ou est-ce plutôt l'absence d'un besoin d'en parler avec nous ou encore de l'absence de détresse? Beaucoup de verbatim d'infirmières ont mentionné que les patients ne désirent pas être aidés, ce qui ne veut pas dire, d'une part, que le dialogue ou l'aide offerte par l'infirmière à ce moment-là n'est pas significatif pour eux. D'autre part, on constate que cette deuxième phase de l'étude met en lumière le caractère introspectif du dépistage qui prédomine dans les thèmes concernant l'utilité de l'outil pour le patient et que cet aspect mérite d'être exploré davantage dans les recherches futures.

L'analyse a aussi mis en évidence que le patient partage avec l'infirmière en grande partie la même compréhension de la finalité de l'outil, soit d'identifier des préoccupations ou des problèmes pouvant interférer avec le bien-être et qui pourraient être davantage accompagnés en termes de soutien émotionnel et de gestion de symptômes.

Clover, Mitchell, Britton et Carter (2014) ont mené une étude afin de comprendre les raisons pour lesquelles les patients traités en ambulatoire qui rapportaient une détresse émotionnelle (4 et plus au TD) refusaient l'aide offerte. Dans cette étude $71 \%$ de leur échantillon ( $\mathrm{N}=311)$ ont refusé cette aide. Notre étude rapporte 77, $3 \%$ de participants qui ne souhaitent pas recevoir de l'aide. Toutefois, ce dernier nombre comprend indifféremment ceux qui atteignent le score clinique et ceux qui ne l'atteignent pas. Les raisons données par les participants à l'étude de Clover et al. sont qu'ils préfèrent gérer ça eux-mêmes (46\%) qu'ils reçoivent déjà de l'aide (24\%) et que leur détresse n'est pas suffisamment sévère (23\%). Ces deux derniers thèmes ont par ailleurs été retrouvés dans plusieurs verbatim des participants de notre étude. Une autre étude récente de la part de Tuinman, Van Nuenen, Hagedoom, et Hoekstra-Weebers (2015) présentent des résultats similaires. Dans cette étude, $67 \%$ des patients ne souhaitent pas 
de référence et un $20 \%$ supplémentaire ont exprimé un peutêtre. ( $\mathrm{N}=1340)$. C'est parmi les gens célibataires que le souhait de recevoir de l'aide est le plus fort. Ils sont deux fois plus susceptibles de désirer une référence que les gens mariés. Létude de Johnnson en 2010 citée par Vanhoose et al., 2015 indique d'ailleurs des taux les plus élevés de détresse parmi les femmes célibataires. Peut-on déceler dans ces résultats, le caractère protecteur pour la santé psychologique de la présence d'un partenaire de vie qui peut offrir soit des interactions positives, un soutien émotionnel ou un soutien social suffisant pour atténuer la détresse (Institut canadien d'information sur la santé, 2012)? Mais alors qui soutient la famille qui elle soutient le patient? La nécessité de dépister la détresse chez les proches aidants se fait de plus en plus sentir au fur et à mesure que le dépistage devient systématique auprès de personnes atteintes de cancer (Direction générale de cancérologie, 2015).

Létude de Clover a aussi révélé que parmi ceux jugeant leur détresse pas suffisamment sévère pour désirer de l'aide, 18 \% atteignaient un score de 8 et plus. D'autres études à ce sujet sont nécessaires. Le stigma associé à l'aide psychologique a été maintes fois décrit, mais la présence de celui-ci influencerait moins négativement lorsqu'il s'agit de cancer (Clover et al., 2013 cités par Clover et al., 2014). Par ailleurs, Salmon, Clark, McGrath et Fisher (2014) nous mettent en garde qu'il peut être préjudiciable au patient de lui dire qu'il a besoin d'aide sur la base de ses réponses à des questions. Ces auteurs rapportent eux aussi les résultats d'études indiquant que parmi ceux qui éprouvent de la détresse émotionnelle, moins de la moitié ont cherché ou ont l'intention de chercher de l'aide. Et moins du quart accepte la référence en oncopsychologie dans une autre étude citée par Salmon et al. (Shimizu et al., 2010). À la lumière de nos résultats et d'autres études, il nous apparaît comme clinicien qu'il ne faut pas cultiver, comme seule réponse possible à un score élevé, la proposition d'une référence, mais en faire un moment fort pour soit souligner une force présente ou refléter un désir légitime chez le patient. Comme le mentionnent ces auteurs, un dépistage positif ne doit pas être interprété comme une obligation ou une attente envers les patients d'accepter une aide formelle. L'analyse de leurs perceptions laisse entrevoir que pour un certain groupe de personnes, le dépistage n'a ni la même valeur d'utilité. Ces pistes demeurent à explorer.

Il ressort aussi à la lumière de nos résultats que la gestion de symptômes a été très peu évoquée dans les commentaires écrits tant chez les participants atteints de cancer que chez les infirmières. Il est à noter que la majorité de nos participants patients de la phase 2 tout comme la phase 1 ont été sollicités en début de parcours, à un moment donc où la charge associée aux symptômes peut être moins élevée (Baba, Fransson, et Lindh, 2007).

Ce type d'échelle, telle que l'ESAS, a été développée afin de faciliter la pratique clinique et de permettre de mesurer des résultats de soins (Baba, Fransson, et Lindh, 2007). Une étude suédoise rapporte tout comme nous que le personnel de soins considère que l'ESAS est un bon instrument pour obtenir de l'information sur les symptômes des patients, mais cette étude indique aussi que selon les infirmières, elle fournit peu d'aide lors des visites médicales (Baba et al., 2007).
Nos résultats indiquent que les symptômes, si on exclut les données manquantes, sont : soit toujours (27,7\%), soit la plupart du temps $(50,5 \%)$ réduits à un niveau où les patients sont confortables. Il reste tout de même, plus d'un patient sur 5 qui sont, au mieux assez souvent confortable. Une personne a indiqué jamais, et 2 rarement. Bien qu'il s'agisse d'un petit nombre de participants, peut-on faire mieux ou l'inconfort est-il inévitable? Maintes fois, nous avons constaté, lors de l'implantation de l'ODD, dans notre établissement, que la gestion de symptômes n'y est pas toujours optimale en ce qui a trait à son évaluation et dans son aspect de documentation afin d'assurer la continuité des soins. Ainsi, bien que le confort physique reçoit de l'attention depuis longtemps en oncologie, il demeure à faire encore dans ce domaine.

Dans un tout autre ordre d'idée, nous avons été étonnés par le pourcentage élevé de participants atteints de cancer $(40,2 \%)$ qui ont rapporté l'absence de discussion avec un professionnel post-complétion de l'outil. Bien qu'une interprétation différente à la question ait pu se produire chez nos participants pour expliquer ce résultat, voir la section sur les limites de l'étude, il nous faut aussi admettre que la discussion souhaitée n'a sans doute pas toujours lieu. Or ce retour est sans nul doute l'aspect le plus crucial et maintes fois souligné dans la formation des professionnels au dépistage (Fillion et al., 2011). Comment expliquer? Le temps ou son corollaire le manque de temps pour le dépistage fait l'objet d'un verbatim spontané chez plus de 4 infirmières sur 10 (42,2\%), sans compter que la grande majorité d'entre elles ont identifié aussi le manque de temps comme élément d'influence négative sur la pratique dans le QPI. Les infirmières l'ont aussi nommé à toutes les étapes d'implantation de l'outil et à toutes les étapes de notre étude. Ce manque de temps est aussi rapporté par différents chercheurs (Absolom et al., 2011; Asselin, 2012, Arantzamendi M. et Kearney N. (2004)., Fillion et al., 2011; Mitchell, Kaar, Coggan, et Herdman, 2008). Ce contexte « d'être toujours pressé » dans lequel s'inscrit le dépistage pour les infirmières peut peut-être expliquer en partie ce résultat comme nous croyons aussi que le manque de temps perçu aussi par la clientèle peut conduire certains patients à choisir de se taire, comme nous portent à le croire certaines observations cliniques. De plus, l'environnement peu intimiste des salles d'administration de la chimiothérapie a été aussi relevé dans d'autres études (Arantzamendi et Kearney, 2004) et les infirmières font partie des professionnels plus sensibles à cet aspect (Absolom et al., 2011).

Notre proposition de modèle théorique sur la signification du dépistage pour les infirmières tend à indiquer que les infirmières sont grandement favorables au dépistage de la détresse, mais encore faut-il qu'il s'inscrive dans des contextes de pratique cohérents avec cette démarche. Cette proposition mérite d'être incluse dans la clinique et la formation, mais elle pourrait être davantage développée par d'autres études.

De plus, cette proposition théorique ne tient pas compte du caractère introspectif du dépistage nommé par les participants patients. Cet apport de connaissance mérite toute notre attention en clinique. 
Notre étude démontre également une moins grande confiance des infirmières à répondre à la détresse et à un fournir un soutien structuré. Ce sont dans les domaines des besoins psychosociaux et émotionnels qu'elles se sentent moins confiantes. D’ailleurs une majorité de références sont dirigées vers l'oncopsychologue $(86,7 \%)$. À ce sujet, Fillion et al., 2011 mentionnent comme élément clé du succès de l'implantation du dépistage la formation adéquate des intervenants.

\section{Limites de la phase 2 de l'étude}

La plus grande limite de cette phase est associée à la non-validation des questionnaires avant le début de l'étude. Même si l'ensemble des membres de l'équipe de recherche avaient pris connaissance des deux questionnaires et qu'il s'était prononcé sur les questionnaires en termes de contenu, formulation des questions, et que ces questionnaires dérivaient de ceux utilisés dans d'autres enquêtes ou études, certains éléments méthodologiques n'avaient pas été anticipés. Par exemple, la question 6 du QPC se lisait comme suit : après avoir complété le questionnaire de dépistage de la détresse, est-ce qu'un professionnel a discuté avec vous de vos réponses? Le choix de réponse était oui ou non, mais sans espace supplémentaire pour l'expression d'une précision ou d'un commentaire. La forte proportion étonnante de non, soit $40 \%$, nous incite à penser que cette question a pu être mal interprétée. Est-ce que par exemple, certains patients pouvaient s'attendre à une discussion avec un autre professionnel que l'infirmière, même si un tel besoin n'était pas exprimé ou identifié suite au dépistage.

Par ailleurs, la question $7 \mathrm{du}$ QPC demandait à la clientèle si on leur avait proposé l'aide qu'il lui fallait pour répondre aux besoins et préoccupations exprimés. Or si le patient n'avait pas le sentiment d'avoir eu un besoin d'aide, ou avoir exprimé une préoccupation particulière, il lui était difficile de répondre avec le choix de réponse proposé soit le oui, soit le non. Heureusement, la plupart des personnes annotaient à côté la difficulté soulevée et dans la section « précisez » nous pouvions comprendre ou interpréter ce qu'il percevait de la situation.

Le QPI comportait aussi une question sujette à interprétation diverse. Ainsi la question is qui interrogeait l'infirmière sur le

\section{RÉFÉRENCES}

Absolom, K., Holch, P., Pini, S., Hill, K., Liu, A., Sharpe, M. Richardson, A., \& Velikova, G. (2011). The detection and management of emotional distress in cancer patients: The views of health-care professionals. Psycho-Oncology, 20, 601-608. doi:10.1002/pon.1916

Admiraal, J.M., van Nuenen, F.M., Burgerhof, J.G.M., Reyners, A.K.L., \& Hoekstra-Weebers, J.E.H.M. (2016). Cancer patients' referral wish: Effects of distress, problems, socio-demographic and illnessrelated variables and social support sufficiency. Psycho-Oncolog, $Y$ 25(11), 1363-1370. doi:10.1002/pon.4067.

Alberta Health Services (2012 A). Evaluation tool: Baseline. Patient perception of care quality. Instrument developed by Teno, J., Boston University, toolkit of instruments adapted by Cancer Care Ontario. nombre de fois où elle a dépisté la détresse en utilisant l'ODD, certaines ont répondu en termes de proportion, soit $75 \%$, I/6 ou $1 / 2$, rendant alors leurs réponses parfois difficiles à juger sur le plan de leur expérience d'utilisation de l'outil. Nous aurions gagné à valider nos questionnaires auparavant. Par ailleurs, le nombre d'infirmières dédiées uniquement au sein étant peu élevé (6), nous devons être excessivement prudents lorsque nous comparons les divers groupes d'infirmières entre elles.

\section{CONCLUSION}

D'une part, les résultats de la phase 1 et 2 de notre étude confirment et précisent différents aspects liés au dépistage de la détresse. Il y a nécessité de proposer le dépistage de la détresse, non seulement parce qu'il permet d'offrir une réponse, mais surtout, il peut nous permettre d'adapter notre offre. Une étude récente (Vanhoose et al., 2015) indique que certains problèmes pèsent plus lourd dans l'apparition de la détresse. Les aspects financiers, les préoccupations et inquiétudes, l'anxiété et les difficultés de sommeil font partie de ces problèmes qui méritent davantage notre attention. Les futures études pourront explorer davantage l'offre d'une plus grande aide dans ces situations. Par ailleurs, nos résultats permettront de poursuivre le travail d'implantation du dépistage dans notre établissement. Il nous faudra ainsi revoir notamment notre compréhension de la finalité de l'outil auprès des deux groupes de participants. La valeur introspective du dépistage pour le patient fait du sens avec leur désir de recourir à leurs ressources personnelles, et ceci pourrait être davantage honoré de la part des professionnels. D'autre part, le souhait de ne pas recevoir de l'aide, mérite d'être davantage approfondi particulièrement chez les hommes. La phase 3 par le biais d'entrevues abordera notamment ce thème.

\section{REMERCIEMENTS}

Cet article présente les résultats ayant trait à la deuxième phase d'une étude à devis mixte séquentiel exploratoire (Creswell et Plano Clarke 2007) portant sur le dépistage systématique de la détresse. Cette recherche a été rendue possible grâce au soutien financier de l'Association canadienne des infirmières en oncologie et de la Fondation de l'hôpital Maisonneuve-Rosemont, fonds administré par le Centre d'excellence en soins infirmiers de cette installation.

Alberta Health Services (2012B). Final Clinician Survey. Modified from BC proof of concept Project with permission. Component of evaluation around confidence with distress assessment and topics adapted from IPODE final course evaluation with permission.

Arantzamendi, M., \& Kearney, N. (2004). The psychological needs of patients receiving chemotherapy: An exploration of nurse perceptions. European Journal of Cancer Care, 13, 23-31. doi:10.1111/j.1365-2354.2003.00438.x

Asselin, P. (2012). Perceptions des infirmières en hémato-oncologie ambulatoire face au dépistage systématique de la détresse et à ses effets sur les soins centrés sur la personne. Présentation faite dans le cadre de la journée scientifique de l'Association québécoise des infirmières en oncologie (AQIO), le vendredi 23 novembre 2012. 
Baba, K., Fransson, P., \& Lindh, J. (2007). Use of a modified ESAS in cancer patients: A pilot study of patient and staff experiences. International Journal of Palliative Nursing, 13(12), 610-616.

Blais, M.C. (2012). Perceptions des infirmières en hémato-oncologie ambulatoire face au dépistage de la détresse. Volet B. Présentation faite dans le cadre de la journée scientifique de l'Association québécoise des infirmières en oncologie (AQIO), le vendredi 23 novembre 2012.

Clover, K.A., Mitchell, A.J., Britton, B., \& Carter, G. (2014). Why do oncology outpatients who reported emotional distress decline help? Psycho-Oncology, 24(7). doi:10.1002/pon.3729

Creswell, J.W., \& Plano Clark, V.L. (2007). Designing and conducting mixed methods research. Thousand Oaks, CA: Sage Publications.

De Marinis, M.G., Piredda, M., Chiara Pascarella, M., Vincenzi, B., Spiga,F., Tartaglini, D., Alvaro, R., \& Matarase, M. (2010). If it is not recorded, it has not been done!? Consistency between nursing records an observed nursing care in an Italian hospital. Journal of Clinical Nursing, 19, 1544-1552 doi:10.1111/j.1365-2702.2009.03012.x

Direction générale de cancérologie (2015). Comité ad hoc sur l'utilisation de dépistage de la détresse pour la clientèle en cancérologie. Diverses communcations personnelles entre membres du comité.

Foss, C., \& Ellefsen, B. (2002). The value of combining qualitative and quantitative approaches in nursing research by means of method triangulation. Journal of Advanced Nursing, 40(2), 242-248.

Fillion, L., Cook, S., Blais, M.-C., Veillette, A.-M., Aubin, M., de Serres, M., et al. (2011). Implementation of screening for distress with professional cancer navigators. Oncologie (Dossier Après Cancer), 13, 277-289. doi:10.1007/s10269-011-2026-8

Institut canadien d'information sur la santé (2012). Le rôle du soutien social dans l'atténuation de la détresse psychologique. https:// secure.cihi.ca/free_products/AiB_ReducingPsychological\%20 DistressFR-web.pdf, pages récupérées le 2 août 2016.

Mitchell, A.J., Kaar, S. Coggan, C., \&, Herdman, J.A. (2008). Acceptability of common screening methods used to detect distress and related mood disorders-preferences of cancer specialists and non-specialists. Psycho-Oncology, 17(3), 226-236. http://onlinelibrary.wiley.com/doi/10.1002/pon.1228

Salmon P., Clark L., McGrath E., \& Fisher P. (2015). Screening for psychological distress in cancer: Renewing the research agenda. Psycho-Oncology, 24(3), 262-268. doi:10.1002/pon.3640

Steele, R., \& Fitch, M.I. (2008). Supportive care needs of women with gynecological cancer. Cancer Nursing, 31(4), 284-291.

Tremblay, N., Roy, O., \& Lecocq, A. (2017). Dépistage systématique de la détresse : portraits et regards croisés sur les perceptions des différents acteurs, perspectives cliniques et de recherche-Partie 1. Revue canadienne de soins infirmiers en oncologie, 27(3), 211-223.

Tuinman, M.A., Van Nuenen, F.M., Hagedoorn, M., \& HoekstraWeebers, J.E.H.M. (2015). Distress, problems and referral wish of cancer patients: Differences according to relationship status and life phase. Psycho-Oncology, 24(6), 699-704. doi:10.1002/pon.3712

VanHoose, L., Black, L.L. Doty, K., Sabata, D., Twumasi-Ankrah, P., Taylor, S., \& Johnson, R. (2015). An analysis of the distress thermometer problem list and distress in patients with cancer. Support care cancer, 23(5), 1225-1232. doi:10.1007/s00520-014-2471-1

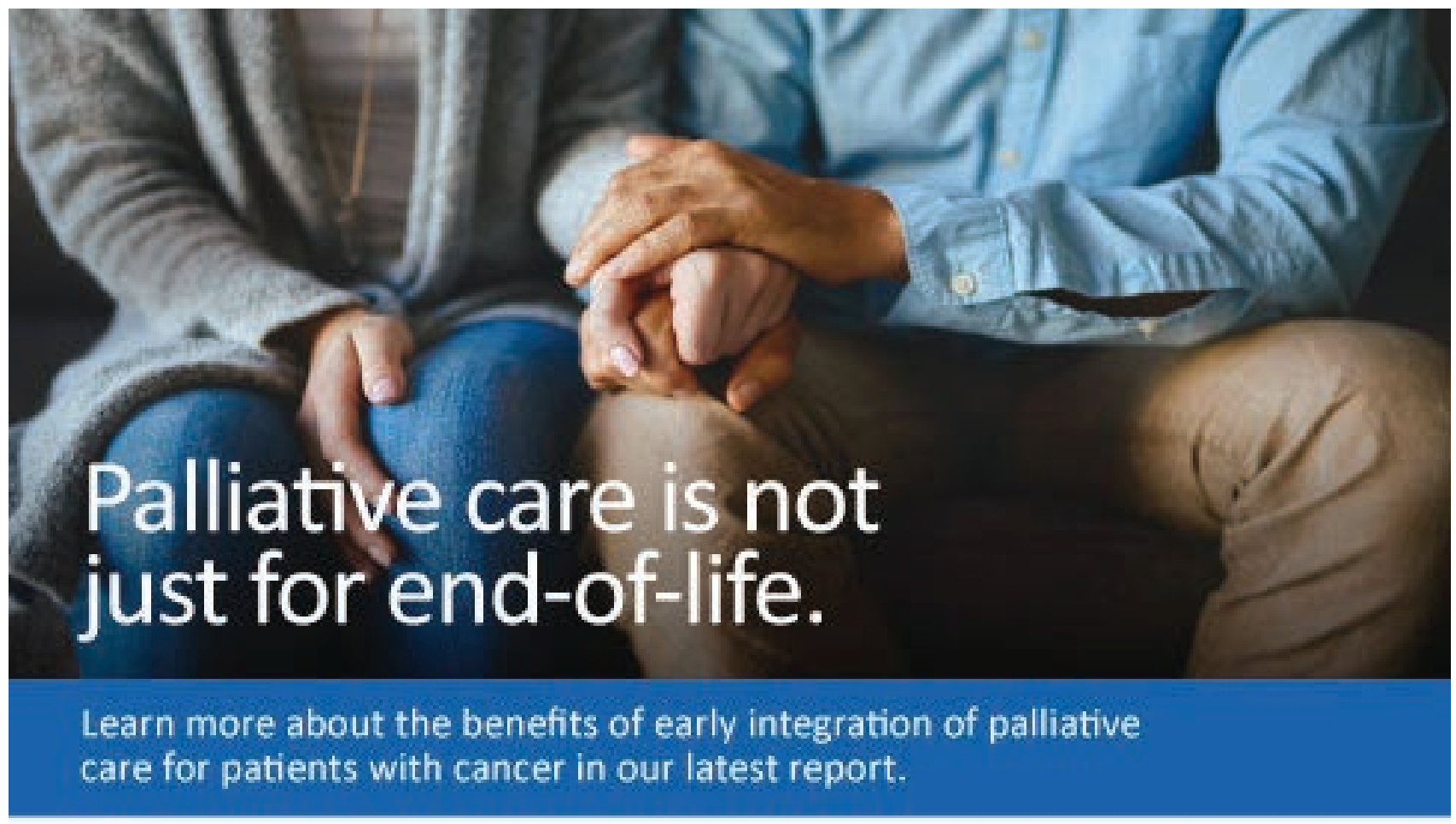

$+$ 